\title{
Escala de Bem-Estar Subjetivo Escolar (EBESE): elaboração e validação de uma medida para avaliação educacional
}

\section{School Subjective Well-Being Scale (EBESE): development and validation of an education assessment measure}

\section{Escala de Bienestar Subjetivo Escolar (EBESE): elaboración y validación de una medida de evaluación educativa}

\author{
João Lucas Dias-Viana ${ }^{1}$, ORCID 0000-0002-7626-3937 \\ Ana Paula Porto Noronha ${ }^{2}$, ORCID 0000-0001-6821-0299 \\ ${ }^{12}$ Universidade São Francisco. Brasil
}

\begin{abstract}
Resumo: O presente estudo teve por objetivo o desenvolvimento da Escala de Bem-Estar Subjetivo Escolar (EBESE) e investigar suas propriedades psicométricas. Realizou-se o processo de elaboração de itens e validação do conteúdo, bem como a realização de estudo de evidências de validade com base na estrutura interna e cálculo dos coeficientes de consistência interna. Participaram 434 estudantes brasileiros, do $7^{\text {a }}$ ano do Ensino Fundamental ao $2^{\circ}$ ano do Ensino Médio, ambos os sexos, com idades entre 12 e 19 anos $(M=14,88 ; D P=1,70)$. Os resultados indicaram, por meio de análises fatoriais exploratórias uma estrutura com três fatores, com índices de ajuste considerados adequados e coeficiente alfa de Cronbach de 0,91. Conclui-se que a EBESE apresentou bons índices de consistência interna e uma estrutura fatorial de acordo com a teoria subjacente. O instrumento tem a sua importância por possibilitar a avaliação dos níveis de bem-estar do aluno, subsidiando intervenções e melhorias no contexto escolar.
\end{abstract}

Palavras-chave: afetos negativos, afetos positivos, testes psicológicos, satisfação com a escola, escola

Abstract: The aim of this study was to develop the Escala de Bem-Estar Subjetivo Escolar - EBESE (School Subjective Well-being Scale - SSWBS) and investigate its psychometric properties. A process of item development, test content validation, study of evidence of validity based on internal structure, and calculation of internal consistency coefficients was carried out. A total of 434 Brazilian students, from the 7th year of Elementary School (7th grade) to the 2nd year of High School (11th grade), of both sexes, between 12 and 19 years of age $(M=14.88 ; S D=1.70)$ participated in the study. Exploratory factor analysis results indicated a three-factor structure, with adequate fit indices and Cronbach's alpha $=0.91$. The SSWBS presented good internal consistency indices and a factor structure in agreement with the underlying theory. The instrument is important because it allows an assessment of student well-being levels, subsidizing interventions and improvements in the school context.

Keywords: negative affects, positive affects, psychological tests, satisfaction with school, school 
Resumen: El objetivo del estudio fue elaborar la Escala de Bem-Estar Subjetivo Escolar EBESE (Escala de Bienestar Subjetivo Escolar) y investigar sus propriedades psicométricas. Inicialmente fue realizado el proceso de construcción de los ítems y la validación de contenido, así como realizar estudio de evidencias de validez con base en la estructura interna y cálculo de los coeficientes de consistencia interna. La muestra contó con 433 estudiantes brasileños que estaban cursando del $7^{\circ}$ año de la Enseñanza Primaria al $2^{\circ}$ año de la Enseñanza Secundaria, varones y niñas, con edades entre 12 y 19 años $(M=14,88 ; D E=$ 1,70). Por medio de análisis factoriales exploratorios, los resultados indicaron una estructura con tres factores con índices de ajuste considerados adecuados y coeficiente alfa de Cronbach de 0,91. Se concluyó que la EBESE presenta índices adecuados de consistencia interna y una estructura factorial de acuerdo con la teoría subyacente. La importancia del instrumento puede ser observada en relación a la posibilidad de evaluación de los niveles de bienestar del alumno, subsidiando intervenciones y mejoras en el contexto escolar.

Palabras clave: afectos negativos, afectos positivos, pruebas psicológicas, satisfacción con la escuela, escuela

Recebido: 28/05/2019

Aceito: $02 / 10 / 2020$

Como citar:

Dias-Viana, J. L. \& Noronha, A. P. (2021). Escala de Bem-Estar Subjetivo Escolar (EBESE): elaboração e validação de uma medida para avaliação educacional. Ciencias Psicológicas, 15(1), e-2349. doi: https://doi.org/10.22235/cp.v15i1.2349

Correspondência: João Lucas Dias-Viana; Universidade São Francisco. E-mail: jolucasviana@gmail.com. Ana Paula Porto Noronha. Universidade São Francisco.E-mail: ana.noronha8@gmail.com

O bem-estar subjetivo (BES) refere-se a como as pessoas avaliam e experienciam as suas vidas em termos de respostas emocionais e julgamentos globais. É um construto multidimensional com estrutura tripartite, composto por um componente cognitivo, a satisfação com a vida, e dois componentes emocionais, os afetos positivos e os afetos negativos (Diener, 1984). O julgamento do quão satisfeito se é com a vida expressa informações diferentes, uma vez que as pessoas não são iguais e estão sob a influência de uma multiplicidade de fatores distintos, como estados de humor, pensamentos e sentimentos presentes no momento (Diener, Lucas, \& Oishi, 2005).

No que se refere ao estudo do BES ao longo do ciclo-vital, pesquisas realizadas confirmaram a estrutura tripartite do modelo de BES em crianças e adolescentes (Diener, 2012; Ortuño-Sierra, Aritio-Solana, Luis, Nalda, \& Fonseca-Pedrero, 2017). No entanto, diferenças quanto aos domínios de satisfação com a vida são observadas. Em adultos, trabalho família, lazer, saúde, finanças, self são os principais domínios que influenciam os níveis de satisfação com a vida. Em populações infantojuvenis, escola, família, amigos, self, 
lazer e ambiente em que vivem são os principais fatores que influenciem os níveis de BES (Huebner, 1991; McCullough, Huebner, \& Laughlin, 2000).

O contexto escolar é considerado um importante fator para o desenvolvimento psicossocial dos sujeitos. Além do desenvolvimento de habilidades acadêmicas, auxilia os alunos a se tornarem mais resilientes diante da adversidade, a sentirem-se mais conectados com as pessoas ao seu redor e a identificarem suas aspirações para o futuro. Não menos importante, a escola é o primeiro lugar onde o indivíduo experimenta a sociedade em todas as suas facetas, e essas experiências podem ter uma profunda influência sobre as atitudes e comportamentos dos alunos na vida (Abed, 2016). Para que cumpra essa função, é importante que o aluno se sinta satisfeito com o seu ambiente escolar e com as relações que estabelece nesse espaço (Alves, Zappe, Patias, \& Dell'Aglio, 2015).

Assim, considerando o seu papel no desenvolvimento psicossocial, a quantidade de anos do processo de escolarização e as horas diárias em que o sujeito permanece na escola, esta caracteriza-se como um importante contexto que influencia no BES de crianças e adolescentes. Tian, Wang e Huebner (2014), embasados na teoria de BES (Diener, 1984), propuseram um modelo teórico de BES escolar, que se refere a como os estudantes avaliam subjetivamente e vivenciam emoções decorrentes das experiências ocorridas na escola. $\mathrm{O}$ modelo inclui um componente cognitivo - satisfação com a escola (SE) - e dois afetivos - os afetos positivos na escola (APE) e afetos negativos na escola (ANE). A SE caracteriza-se pelos julgamentos e avaliações que os alunos fazem das experiências escolares. Os afetos, semelhante ao modelo geral BES, refere-se à frequência com que os alunos experienciam emoções positivas e negativas no contexto escolar (King, Huebner, Suldo, \& Valois, 2007).

Desse modo, faz-se necessário intensificar as discussões e investigações de temas relativos ao bem-estar do aluno quanto à escola, uma vez que pesquisas sobre esta variável contribuem para a promoção de relações saudáveis, engajamento dos alunos, prevenção da evasão escolar e contribuem para os níveis de BES global (Cintra \& Guerra, 2017; Whitley et al., 2012). Além disso, garantir o bem-estar do aluno caracteriza-se como uma das responsabilidades das instituições escolares (Ministério da Educação e Cultura [MEC], 2013). Nesse sentido, considerando a importância das avaliações educacionais de largaescala na avaliação e monitoramento das redes de ensino, espera-se que os estudos de construção e o desenvolvimento de um instrumento de BES escolar, auxilie na produção de indicadores que possibilitem a melhoria de redes de ensino e auxilie na formulação de políticas públicas educacionais com foco no bem-estar do aluno.

Considerando que o avanço do conhecimento acerca de um fenômeno depende da existência de instrumentos de medida válidos e confiáveis, Dias-Viana e Noronha (2020) realizaram uma revisão da literatura com o objetivo de identificar instrumentos que se propusessem a mensuração do BES escolar. Os resultados indicaram que a maior parte das pesquisas utilizaram medidas globais, ou mensuram apenas o componente de satisfação com a escola. O único instrumento encontrado foi o Brief Adolescent's Subjective Well-Being in School Scale (BASWBSS; Tian et al., 2014), o qual possui dois fatores, um que mensura a satisfação com a escola, e outro os afetos na escola. Mesmo que a BASWBSS possua dois itens para a avaliação dos afetos (um para APE e outro para ANE), de acordo com a teoria de BES que embasa a escala, afetos positivos e negativos não são polaridades de uma mesma dimensão, mas são independentes.

Ainda que não exista consenso sobre a quantidade ideal de itens que o fator de um instrumento psicológico deve possuir, espera-se que um fator possua, no mínimo, três itens (Pasquali, 2001). Medidas de item único não conseguem abordar construtos multidimensionais e complexos, limitando a possibilidade de mensuração e comprometendo 
a precisão do instrumento (Konrath, Meier, \& Bushman, 2014). Além disso, os afetos positivos são importantes variáveis que contribuem para a vivência escolar, relacionados com o engajamento e sucesso escolar (Sellstrom \& Bremberg, 2006), e coping (Reschly, Huebner, Appleton, \& Antaramian, 2008). Por outro lado, afetos negativos correlacionamse com evasão escolar, comportamento antissocial e abuso de substâncias (Roeser, 2001). Assim, identifica-se como limitação da BASWBSS o fato de o instrumento possuir um único item para avaliação dos fatores afetivos do BES escolar. Além disso, ressalta-se que um instrumento com maior número de itens permitiria uma melhor mensuração e compreensão da importância dos afetos para a escolarização. Desse modo, este trabalho tem como objetivo apresentar o processo de construção da Escala de Bem-Estar Subjetivo Escolar (EBESE), bem como apresentar evidências de validade com base no conteúdo e na estrutura interna do instrumento.

\section{Método}

Para a construção dos itens foi realizada uma revisão de literatura com base nos três fatores que compõem o BES escolar: satisfação com a escola, afetos positivos na escola e afetos negativos na escola. As informações obtidas a partir do levantamento na literatura disponível, subsidiaram os proponentes desta pesquisa no processo de elaboração de 70 itens iniciais para a EBESE. O conceito teórico utilizado para a elaboração dos itens foi o de bemestar subjetivo desenvolvido por Diener (1984) e o modelo de BES Escolar proposto por Tian et al. (2014). Ressalta-se que a elaboração dos itens seguiu as diretrizes da International Test Comission, que preconiza sobre a construção de itens claros e objetivos, que expressem uma única ideia; que sejam inteligíveis para a população-alvo; consistente com o fator que se pretende avaliar e que apresente validade de face (International Test Comission [ITC], 2018).

\section{Estudo 1 - Evidências de validade baseadas no conteúdo}

\section{Participantes}

Participaram da análise de juízes três psicólogos, ambos os sexos, com título de graduação, mestrado e doutorado em Psicologia. Os participantes possuíam tempo médio de 18 anos de atuação profissional, e conhecimento e experiência em pesquisas nas áreas da psicologia escolar e em avaliação psicológica, bem como em construção de instrumentos. Para o estudo piloto, participaram 20 adolescentes, ambos os sexos, com idades variando de 12 a 13 anos $(M=12,15 ; D P=0,36)$, estudantes do $7^{\circ}$ ano do Ensino Fundamental de uma escola pública do Estado do Ceará, Brasil. Alunos que possuíssem comprometimentos cognitivos severos e/ou algum transtorno que os impossibilitasse de participar da pesquisa foram excluídos da amostra. As informações de quais estudantes se enquadrariam no critério de exclusão da amostra foram obtidas junto à coordenação da escola. Mediante consulta, constatou-se que na instituição escolar não havia alunos que atendiam aos critérios de exclusão da pesquisa.

\section{Instrumentos}

Formulário de Avaliação para Juízes Independentes. No formulário enviado aos três juízes especialistas constaram perguntas de identificação pessoal e de formação. Além disso, havia uma breve descrição da pesquisa e seus objetivos, bem como do construto - BES 
escolar. Na sequência, as instruções de como proceder com a avaliação dos itens, que foram analisados quanto à clareza da linguagem, relevância teórica, pertinência prática e a dimensão teórica.

Escala de Bem-Estar Subjetivo Escolar - versão inicial. O instrumento tem por objetivo avaliar a satisfação do aluno com a escola e a frequência de afetos positivos e negativos vivenciados por estudantes no contexto escolar. Foi composto por duas subescalas. A primeira continha 10 itens iniciais que objetivavam avaliar o fator de satisfação com a escola (aspecto cognitivo do BES escolar), avaliando o polo positivo e negativo do construto, com chave de resposta em escala Likert, variando de 1 (discordo totalmente) a 5 (concordo totalmente). São exemplos de itens: "Estou satisfeito com a minha escola"; "Minha escola é excelente". A segunda subescala, afetos positivos e negativos vivenciados pelos alunos no ambiente escolar, foi composta por 60 itens iniciais, 30 referentes aos afetos positivos e 30 aos afetos negativos. Os itens consistiam em adjetivos, com chave de resposta em escala Likert de cinco pontos, variando de 1 (nunca) a 5 (sempre). São exemplos de itens: "Confiante", "Motivado(a)", "Cansado(a)" e "Zangado(a)". Para avaliação da compreensão dos itens pela população-alvo, foram inseridas as seguintes perguntas: "Teve alguma palavra que você não conhecia?"; "Alguma questão (item) não ficou clara para você, se sim, qual foi (Indique o número da questão)?”; "As instruções para o preenchimento do teste ficaram claras para você?"; "Você teria alguma sugestão sobre as questões ou para o teste no geral?".

\section{Procedimentos}

Inicialmente, foi solicitada a autorização junto à direção de uma escola pública do Estado do Ceará, Brasil, para a aplicação do instrumento. Em seguida, o projeto de pesquisa foi submetido à apreciação por um Comitê de Ética em Pesquisa de uma universidade brasileira, e aprovado com o número CAAE: 97953218.2.0000.5514. Após aprovação, um e-mail foi enviado aos juízes especialistas convidando-os a participarem da pesquisa e a avaliarem os itens. A concordância em participar do estudo ocorreu por meio da assinatura do Termo de Consentimento Livre e Esclarecido. (TCLE) e um link de acesso ao protocolo de avaliação dos itens foi enviado aos pesquisadores especialistas.

Após a análise de juízes, na etapa do estudo piloto, os participantes tiveram o Termo de Consentimento Livre e Esclarecido (TCLE) assinado por um dos pais ou responsáveis legais, além do Termo de Assentimento Livre e Esclarecido (TALE) assinado pelo próprio aluno, respeitando os aspectos éticos exigidos para pesquisas envolvendo seres humanos. A aplicação do instrumento ocorreu de forma presencial e coletiva, em horário previamente agendado com a escola, abarcando uma turma de alunos. Ressalta-se que a versão da EBESE utilizada no estudo piloto foi decorrente da análise de juízes, em que itens foram excluídos por não atingirem os critérios estabelecidos de validade de conteúdo. Durante a aplicação, os alunos receberam as devidas orientações quanto à forma de como os instrumentos deveriam ser preenchidos. O tempo para a aplicação da versão piloto da escala foi de 30 minutos. Após finalizarem o preenchimento do instrumento, o pesquisador realizou um levantamento das possíveis dúvidas que surgissem em decorrência do processo de resposta.

\section{Análise de Dados}

As pontuações e respostas dos juízes ao Formulário de Avaliação para Juízes Independentes foram armazenadas em uma planilha eletrônica e foram analisadas de acordo com o Coeficiente de Validade de Conteúdo (CVC; Hernández-Nieto, 2002). Foram mantidos os itens com CVC igual ou maior a 0,80. 
No estudo piloto, foram utilizadas estatísticas descritivas para caracterização da amostra por meio do programa estatístico Statistical Package for the Social Sciences (SPSS), v.25. As respostas dos 20 alunos ao questionário de avaliação da EBESE foram lidas na íntegra e analisadas qualitativamente, agrupadas segundo a semelhança de seus conteúdos, utilizando a metodologia de análise de conteúdo proposta por Bardin (2011). As dúvidas e sugestões verbalizadas pelos alunos durante a aplicação foram anotadas para posterior apreciação.

\section{Resultados do Estudo 1}

Os 70 itens construídos para versão inicial da EBESE foram submetidos à análise de juízes e foram mantidos os itens com CVC igual ou maior que 0,80 . Assim, para o fator de satisfação com a escola, três itens foram excluídos: "Quando meus amigos falam de suas escolas, penso que a escola deles é melhor do que a minha" $(0,76)$; "Minha escola está próxima de um modelo de escola ideal" $(0,36)$ e "Na maioria dos aspectos, estou satisfeito com a minha escola" $(0,78)$. Sete itens de afetos positivos na escola foram excluídos: "aceito(a)" (0,76), “aliviado(a)" (0,63), “bem" (0,76), “confortável” $(0,76)$, "esperançoso(a)" $(0,76)$, “forte" $(0,63)$ e "otimista" $(0,69)$. Onze itens de afetos negativos foram excluídos: "ameaçado(a)" $(0,76)$, "assustado(a)" $(0,76)$, "culpado(a)" $(0,69)$, "deprimido(a)" $(0,76)$, "entediado(a)" $(0,76)$, "humilhado(a)" $(0,76)$, "incompreendido(a)" $(0,76)$, “magoado(a)" (0,69), “amedrontado(a)" (0,69), "perturbado(a)" $(0,63)$ e "envergonhado(a) $(0,76)$ ". Desta forma, 21 itens foram excluídos da escala.

Com a versão de 49 itens da EBESE foi realizado o estudo piloto. Os participantes não manifestaram dúvidas quanto às instruções, forma de resposta e compreensão dos itens. Apenas o item "entusiasmado(a)" foi assinalado como de difícil compreensão o que ocasionou sua remoção da escala. Assim, após análise de juízes e estudo piloto, foram removidos 22 itens, o que resultou em uma versão da EBESE composta por 48 itens.

\section{Estudo 2 - Evidências de validade baseadas na estrutura interna}

\section{Participantes}

Participaram deste estudo uma amostra composta por 434 alunos, com predomínio do sexo feminino $(n=240 ; 55,30 \%)$, idades entre 12 e 19 anos $(M=14,88 ; D P=1,70)$, estudantes do $7^{\circ}$ ano do Ensino Fundamental ao terceiro ano do Ensino Médio, regularmente matriculados em uma escola pública do Estado do Ceará, Brasil. Alunos que possuíssem comprometimentos cognitivos severos e/ou algum transtorno que o impossibilitasse de participar seriam excluídos da amostra. Mediante consulta à coordenação da escola, constatou-se que na instituição escolar não havia alunos que atendessem aos critérios de exclusão desta pesquisa.

\section{Instrumentos}

Questionário de Identificação Sociodemográfica e Escolar. Instrumento desenvolvido pelos proponentes desta pesquisa. Composto por questões relativas à idade, sexo, escola e ano escolar.

Escala de Bem-Estar Subjetivo Escolar. Instrumento elaborado para avaliação do bem-estar do aluno no contexto escolar. A versão utilizada nesta etapa resulta do estudo de validação do conteúdo (Estudo 1). Assim, utilizou-se uma versão da EBESE composta por 
48 itens, distribuídos em três fatores: Satisfação com a Escola (7 itens), Afetos Negativos na Escola (22 itens) e Afetos Positivos na Escola (19 itens).

\section{Procedimento}

A participação dos alunos com idades inferiores a 18 anos na pesquisa ocorreu mediante a entrega do Termo de Consentimento Livre e Esclarecido assinado por um dos pais ou responsáveis legais, além do Termo de Assentimento Livre e Esclarecido assinado pelo próprio estudante. Alunos com idade igual ou superior a 18 anos participaram da pesquisa mediante assinatura do TCLE. A aplicação do instrumento ocorreu de forma presencial e coletiva, em horário previamente agendado com a escola.

\section{Análise de Dados}

Inicialmente, foi testado se a matriz de dados da EBESE era passível de fatoração por meio do cálculo do índice Kaiser-Meyer-Olkin (KMO) e pelo Teste de Esfericidade de Bartlett. Valores de KMO iguais ou superiores a 0,70 e com teste de esfericidade com níveis de significância $p<0,05$ indicam que a matriz é fatorável, sendo possível a aplicação de Análise Fatorial Exploratória (Pasquali, 1999). Em seguida, para definição de números de fatores da EBESE foram utilizados o método de análises paralelas e o Minimum Average Partial (MAP), análise paralela e método de Hull (Lorenzo-Seva, Timmerman, \& Kaers, 2011; Velicer, 1976).

Em seguida, os parâmetros da solução fatorial foram estimados por meio de rotação Oblimin e estimador dos mínimos quadrados ponderados (Weighted Least Squares Mean and Variance-adjusted - WLSMV), a partir da matriz de correlações policóricas entre as variáveis. Esse estimador é considerado robusto e recomendado para itens de natureza categórica e ordinal, como escalas Likert. Tendo em vista que a proposta da EBESE é a de um instrumento breve, foram mantidos os itens de discriminação moderada e alta, com cargas fatoriais igual ou superior a $0,40 \mathrm{em}$ pelo menos um dos fatores. Em seguida, realizouse Análise Fatorial Exploratória, e foram considerados os seguintes índices de ajuste: RootMean-Square Error of Approximation (RMSEA; valor de referência < 0,08), Comparative Fit Index (CFI, valor de referência >0,90) e Tucker-Lewis Index (TLI; valor de referência > 0,90; Hair, Black, Babin, Anderson, \& Tatham, 2009)

Os índices de consistência interna da escala geral e para cada um dos seus fatores foram calculados por meio do alfa de Cronbach e ômega de McDonald. Valores de coeficientes de consistência interna iguais ou superiores a 0,70 são considerados adequados (Campo-Arias \& Oviedo, 2008). Também foram utilizadas curvas de informação do teste, pois são mais específicas que os coeficientes tradicionais de fidedignidade, permitindo identificar a precisão do instrumento em cada parte do traço latente. A estrutura interna da EBESE também foi analisada a partir da Teoria de Resposta Item, utilizando-se o modelo de Rasch (Bond \& Fox, 2015) para investigação dos parâmetros de dificuldade dos itens e das pessoas. São considerados adequados itens que apresentem correlações item-pessoas acima de 0,30. Também foi investigado o ajuste dos itens por meio dos índices de Infit e Outfit, tomando como referência os valores situados entre o intervalo de 0,5 a 1,5 (Linacre, 2014). As análises deste estudo foram realizadas com a utilização do software Factor Analysis 10.8, Mplus 7.11, RStudio e Winsteps 3.74. 


\section{Resultados do Estudo 2}

Inicialmente, constatou-se um coeficiente Kaiser-Meyer-Olkin (KMO) de 0,94, que sugere um nível suficiente de variância comum entre os itens para realização de análise fatorial. O Teste de Esfericidade de Bartlett, que avalia a significância geral de todas as correlações em uma matriz de dados, apresentou significância estatística $(p<0,001)$. Tais dados, indicam a possibilidade de fatoração dos dados. Quanto à solução fatorial da EBESE, os métodos de retenção não foram unânimes, com recomendações de soluções de um (Método de Hull), três (MAP) e até quatro fatores (Análise Paralela).

Ao analisar estas soluções, observou-se 19 itens que apresentaram cargas cruzadas acima de 0,30 e um item que não carregou em nenhum dos fatores. Assim, optou-se pela exclusão desses itens, considerando que o objetivo consistia em desenvolver uma medida psicométrica breve e de melhor qualidade. Foram considerados os itens com cargas fatoriais iguais ou superiores a 0,40 na dimensão teórica esperada (Hair et al., 2009). Desse modo, 20 itens foram excluídos, restando uma versão da EBESE contendo 28 itens.

Com a versão de 28 itens da EBESE, foi realizada análise fatorial exploratória para investigar os índices de ajuste do modelo com um, três e quatro fatores, utilizando-se os índices RMSEA, CFI e TLI. A solução com um fator foi a que apresentou piores ajustes ( $\mathrm{RMSEA}=0,13 ; \mathrm{CFI}=0,74 ; \mathrm{TLI}=0,72$ ). Os dados evidenciaram que a solução com quatro fatores apresentou melhores índices (RMSEA $=0,05 ; \mathrm{CFI}=0,97 ; \mathrm{TLI}=0,96$ ) quando comparado à solução com três (RMSEA $=0,06 ; \mathrm{CFI}=0,95 ; \mathrm{TLI}=0,94)$. No entanto, a interpretabilidade dos valores assumidos pelas cargas fatoriais não permitiu a identificação de um padrão teórico de distribuição, que justificasse um quarto fator. Dessa forma, os resultados favoreceram um modelo com três fatores, cujos itens, cargas e coeficientes de consistência interna são apresentados na Tabela 1. 
Tabela 1

Resultados da análise fatorial exploratória (rotação oblimin) e coeficientes de consistência interna

\begin{tabular}{|c|c|c|c|}
\hline Item & $\mathrm{F} 1$ & $\mathrm{~F} 2$ & F3 \\
\hline 1. A escola é um lugar no qual me sinto bem & $\mathbf{0 , 4 1}$ & $-0,12$ & 0,27 \\
\hline 2. Minha escola é ruim & $-0,68$ & 0,01 & $-0,03$ \\
\hline 3. Estou insatisfeito com a minha escola & $-0,66$ & 0,08 & 0,06 \\
\hline $\begin{array}{l}\text { 4. Se eu tivesse a oportunidade de escolher uma escola para estudar, escolheria a } \\
\text { minha }\end{array}$ & 0,66 & 0,10 & $-0,01$ \\
\hline 5. Estou satisfeito com a minha escola & $\mathbf{0 , 8 4}$ & $-0,02$ & 0,00 \\
\hline 6. Minha escola é excelente & $\mathbf{0 , 7 2}$ & 0,08 & 0,06 \\
\hline 7. Tem muitas coisas que gosto na minha escola & $\mathbf{0 , 6 0}$ & 0,01 & 0,05 \\
\hline 8. Aborrecido & 0,00 & 0,64 & $-0,19$ \\
\hline 9. Agitado & 0,18 & 0,42 & 0,29 \\
\hline 10. Ansioso & 0,23 & 0,48 & $-0,13$ \\
\hline 11. Cansado & $-0,01$ & 0,44 & $-0,23$ \\
\hline 12. Chateado & $-0,03$ & 0,59 & $-0,26$ \\
\hline 13. Furioso & $-0,02$ & $\mathbf{0 , 8 8}$ & 0,04 \\
\hline 14. Impaciente & $-0,05$ & 0,61 & 0,02 \\
\hline 15. Irritado & $-0,01$ & $\mathbf{0 , 8 7}$ & $-0,05$ \\
\hline 16. Com Raiva & $-0,05$ & 0,79 & 0,00 \\
\hline 17. Tenso & 0,11 & $\mathbf{0 , 5 7}$ & $-0,22$ \\
\hline 18. Zangado & 0,03 & 0,95 & 0,01 \\
\hline 19. Capacitado & 0,12 & $-0,04$ & 0,68 \\
\hline 20. Cheio de Energia & 0,19 & 0,01 & 0,56 \\
\hline 21. Competente & 0,04 & 0,02 & 0,72 \\
\hline 22. Compreendido & 0,24 & $-0,09$ & 0,46 \\
\hline 23. Confiante & 0,00 & $-0,04$ & 0,75 \\
\hline 24. Corajoso & $-0,02$ & 0,05 & 0,67 \\
\hline 25. Determinado & $-0,03$ & 0,18 & $\mathbf{0 , 8 5}$ \\
\hline 26. Enturmado & 0,17 & 0,02 & $\mathbf{0 , 4 5}$ \\
\hline 27. Interessado & 0,16 & 0,07 & 0,56 \\
\hline 28. Motivado & 0,25 & $-0,05$ & 0,62 \\
\hline Coeficiente Alpha de Cronbach & 0,82 & 0,88 & 0,87 \\
\hline Coeficiente Ômega de McDonald & 0,87 & 0,90 & 0,89 \\
\hline
\end{tabular}

Nota. F1 = satisfação com a escola; $\mathrm{F} 2$ = afetos negativos na escola, $\mathrm{F} 3=$ afetos positivos na escola 
Como pode ser observado na Tabela 1, a solução com três fatores apresentou cargas cruzadas de baixa magnitude $(\leq|0,29|)$. A interpretabilidade das cargas fatoriais e do padrão de distribuição dos itens no fator corroboraram o modelo teórico tripartite de bem-estar subjetivo. $\mathrm{O}$ primeiro fator explicou itens relacionados à satisfação do aluno com a escola, a avaliação que cognitiva que o aluno faz de sua escola. $\mathrm{O}$ fator 2 e o fator 3 explicaram itens relacionados aos afetos positivos e negativos experienciados pelos alunos no ambiente da escola. As correlações entre os fatores foram de moderada magnitude $\left(r_{\mathrm{F} 1 \times \mathrm{F} 2}=-0,39 ; r_{\mathrm{F} 1 \times \mathrm{F} 3}\right.$ $=0,43$ e $r_{\mathrm{F} 2 \times \mathrm{F} 3}=-0,34$ ), indicando uma relação oblíqua entre as variáveis (Damásio, 2012).

O cálculo dos índices de consistência interna, evidenciaram coeficientes alfa de Cronbach variando de 0,82 a 0,88, e ômegas de 0,87 a 0,90. Em seguida, foram calculados os índices de consistência para versão da EBESE com 28 itens que resultaram em uma alpha de 0,91 e ômega de McDonald de 0,93, indicando bons índices de precisão para o instrumento.

A etapa seguinte consistiu em investigar com maior precisão a fidedignidade dos três fatores por meio das curvas de informação, utilizando-se TRI. Estas análises permitam constatar que o primeiro fator, satisfação com a escola, apresentou um nível de precisão mais alto para o intervalo entre -2 e 0 da escala de teta. Afetos negativos na escola, segundo fator da EBESE, apresentou maiores níveis de precisão no intervalo entre $-1 \mathrm{e}+3 \mathrm{a}$, evidenciando maior nível de informação sobre amplo espectro do traço latente, com maior precisão para a população situada acima da média da população (estandardizada como 0). Constatou-se que o fator 3, afetos positivos na escola, apresentou maior precisão no intervalo entre $-2 \mathrm{e}+1$, evidenciando um melhor funcionamento do instrumento no polo mais baixo do traço latente. A análise das curvas de informações permite constatar que o instrumento possui maior confiabilidade na mensuração de alunos com menores níveis de satisfação com a escola, de afetos positivos e maiores níveis de afetos negativos (ver Figura 1). 

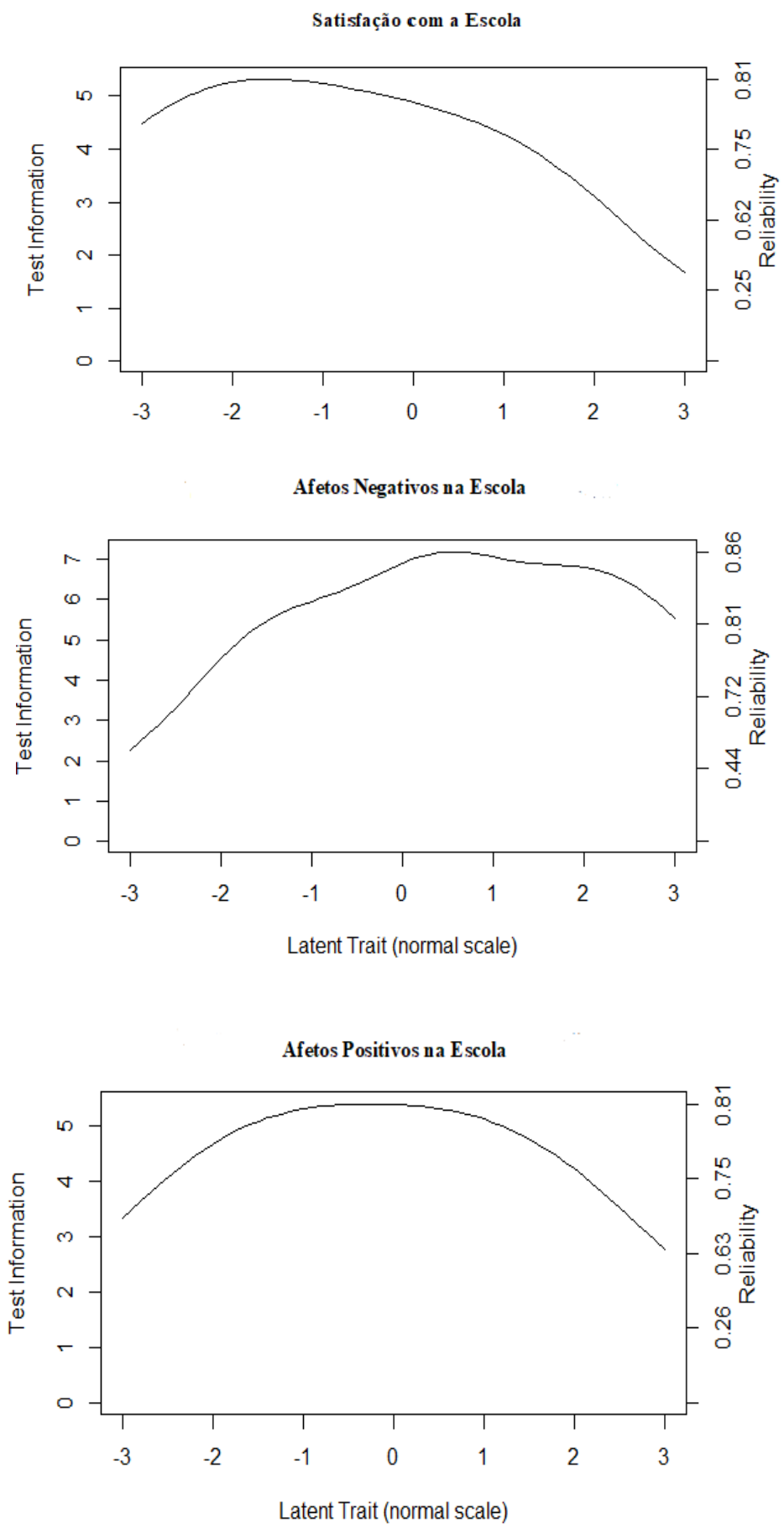

Figura 1.

Curvas de informação dos fatores da EBESE-28 itens. 
Na sequência foi usado o modelo de Teoria de Resposta ao Item (TRI) por meio da análise de Rating Scale (ver Tabela 2). Todos os fatores foram avaliados como unidimensionais, de acordo com os critérios de Linacre (2014), em que a variância do primeiro contraste deve ser menor que o segundo. A análise da Tabela 2, evidencia que a maioria dos itens estão dentro do ajuste esperado, valores infit e outfit situados no intervalo entre 0,5 e 1,5 (Linacre, 2014). Apenas o item 9 ("agitado(a)") ficou com ajustes fora do esperado.

Em relação às correlações item-total, o item 9 ("agitado (a)"), apresentou menor coeficiente $(r=0,35)$ e índices de infit e outfit fora do intervalo de referência, indicando que este item não representa tão bem o construto avaliado. Uma nova análise fatorial exploratória foi realizada com os 27 itens restantes e observou-se melhoria nos índices de ajuste da escala, com $\mathrm{CFI}=0,96$; $\mathrm{TLI}=0,95 ; \mathrm{RMSEA}=0,06$. Quanto aos demais itens, as correlações variaram de 0,50 a 0,81 , interpretadas de moderadas a altas. A consistência interna dos itens foi considerada como adequada, com menor valor de 0,96 (F3) e maior valor de 0,99 (F1 e F2). Em relação às pessoas, os coeficientes foram de 0,78 (F1), 0,84 (F2) e 0,83 (F3), valores também considerados adequados. No que diz respeito à dificuldade dos itens, os itens 6 de satisfação com a escola ("Minha escola é excelente"), 13 afetos negativos na escola ("furioso(a)"), e 22 de afetos positivos na escola ("compreendido(a)") foram os menos endossados. Os itens 2 de SE ("minha escola é ruim"), 11 de ANE ("cansado(a)"), e 26 de APE ("enturmado(a)") os de maior facilidade de endosso. 
Tabela 2

Análise dos itens da EBESE pelo modelo de Rasch para os três fatores da EBESE

\begin{tabular}{|c|c|c|c|c|c|}
\hline $\begin{array}{c}\text { Itens } \\
\text { do } \\
\text { Fator } 1\end{array}$ & Escore Bruto & Dificuldade do Item (b) & Infit & Outfit & $\begin{array}{l}\text { Correlação item- } \\
\text { theta }\end{array}$ \\
\hline 1 & 1435 & 0,29 & 1,19 & 1,19 & 0,60 \\
\hline 2 & 1731 & $-1,38$ & 0,91 & 0,89 & 0,67 \\
\hline 3 & 1555 & $-0,10$ & 1,11 & 1,22 & 0,66 \\
\hline 4 & 1353 & 0,61 & 1,10 & 1,14 & 0,69 \\
\hline 5 & 1465 & 0,08 & 0,70 & 0,69 & 0,80 \\
\hline 6 & 1331 & 0,67 & 0,95 & 0,94 & 0,72 \\
\hline 7 & 1551 & $-0,17$ & 1,02 & 1,06 & 0,66 \\
\hline Média & 1488,7 & 0,00 & 1,00 & 1,02 & - \\
\hline$D P$ & 127,5 & 1,00 & 0,15 & 0,18 & - \\
\hline $\begin{array}{c}\text { Itens } \\
\text { do } \\
\text { Fator } 2 \\
\end{array}$ & Escore Bruto & Dificuldade do Item (b) & Infit & Outfit & $\begin{array}{l}\text { Correlação item- } \\
\text { theta }\end{array}$ \\
\hline 8 & 1329 & $-0,28$ & 0,88 & 0,87 & 0,71 \\
\hline 9 & 1360 & $-0,39$ & 1,85 & 1,98 & 0,34 \\
\hline 10 & 1429 & $-0,73$ & 1,35 & 1,50 & 0,50 \\
\hline 11 & 1583 & $-1,13$ & 1,21 & 1.19 & 0,57 \\
\hline 12 & 1126 & 0,37 & 0,90 & 0,90 & 0,69 \\
\hline 13 & 923 & 0,92 & 0,71 & 0,66 & 0,76 \\
\hline 14 & 1289 & $-0,14$ & 1,05 & 1,14 & 0,65 \\
\hline 15 & 1076 & 0,48 & 0,60 & 0,60 & 0,81 \\
\hline 16 & 1175 & 0,19 & 0,72 & 0,74 & 0,77 \\
\hline 17 & 1241 & 0,01 & 1,03 & 1,04 & 0,66 \\
\hline 18 & 1005 & 0,71 & 0,60 & 0,59 & 0,81 \\
\hline Média & 1230,5 & 0,00 & 0,99 & 1,02 & - \\
\hline$D P$ & 185,1 & 0,59 & 0,36 & 0,41 & - \\
\hline $\begin{array}{c}\text { Itens } \\
\text { do } \\
\text { Fator } 3 \\
\end{array}$ & Escore Bruto & Dificuldade do Item (b) & Infit & Outfit & $\begin{array}{l}\text { Correlação item- } \\
\text { theta }\end{array}$ \\
\hline 19 & 1288 & 0,24 & 0,83 & 0,83 & 0,72 \\
\hline 20 & 1321 & 0,07 & 1,1 & 1,19 & 0,67 \\
\hline 21 & 1350 & $-0,01$ & 0,89 & 0,9 & 0,71 \\
\hline 22 & 1191 & 0,70 & 1,07 & 1,06 & 0,64 \\
\hline 23 & 1333 & 0,00 & 0,83 & 0,83 & 0,74 \\
\hline 24 & 1299 & 0,17 & 1,1 & 1,1 & 0,64 \\
\hline 25 & 1405 & $-0,24$ & 0,87 & 0,86 & 0,72 \\
\hline 26 & 1483 & $-0,51$ & 1,32 & 1,4 & 0,58 \\
\hline 27 & 1446 & $-0,49$ & 1,12 & 1,18 & 0,61 \\
\hline 28 & 1313 & 0,07 & 0,82 & 0,81 & 0,74 \\
\hline Média & 1342,9 & 0,00 & 0,99 & 1,02 & - \\
\hline$D P$ & 79,8 & 0,34 & 0,16 & 0,19 & - \\
\hline
\end{tabular}

Nota. Fator 1 = Satisfação com a escola; Fator 2 = Afetos negativos na escola; Fator 3 = Afetos positivos na escola 


\section{Discussão}

Esta pesquisa teve como objetivo propor um modelo de uma medida para mensuração do bem-estar subjetivo escolar e investigar as propriedades psicométricas do instrumento. Para atender este objetivo, realizou-se estudo de validade de conteúdo para os itens da escala, análise fatorial exploratória e TRI. Os resultados indicaram que a EBESE apresentou evidências de validade com base no conteúdo e na estrutura interna, além de coeficientes de fidedignidade adequados, reunindo dados empíricos iniciais para utilização da escala para avaliação do BES escolar. A análise de estrutura interna permitiu identificar que a solução fatorial mais adequada foi a de três fatores.

A análise fatorial dos itens permitiu chegar a uma solução fatorial composta por três fatores, sendo a mais interpretável e com índices de ajuste considerados adequados. Chegouse a uma versão da EBESE de 27 itens, distribuídos em três fatores, a saber, satisfação com a escola ( 7 itens), afetos positivos na escola (10 itens) e afetos negativos na escola (10 itens). Em relação à estrutura fatorial da EBESE, foi possível corroborar os pressupostos teóricos propostos por Diener (1984) e Tian et al. (2014), com um modelo tripartite, composto por uma dimensão de avaliação cognitiva (satisfação com a escola) e duas de avaliação afetiva (afetos positivos na escola e afetos negativos na escola).

No que diz respeito ao ajuste dos itens do modelo por meio de Rating Scale, apenas o item 9 ("agitado(a)") apresentou valores infit e outfit fora do esperado. O desajuste do item no infit indica que o item não está relacionado com o traço latente, indicando que deve ser removido da escala. Desajustes no outfit indicam o endosso do participante ao item, independentemente do nível de habilidade (Linacre, 2014). A partir de uma análise qualitativa desse item, compreende-se que agitação/agitado(a), embora tenha repercussões afetivas, está mais ligado ao campo comportamental e psicomotor do que propriamente afetivo. Além disso, a relativa facilidade de endosso dos alunos a este item, independentemente do nível de habilidade evidencia o quão típico é esse comportamento no período da adolescência e caracteriza-se como uma das principais queixas escolares (Carneiro \& Coutinho, 2015). Assim, justifica-se um endosso independentemente do nível de habilidade do estudante, pois representa um comportamento típico da etapa do desenvolvimento e do contexto.

Quanto à facilidade e dificuldade de endosso dos itens, aspectos tanto do contexto escolar, quanto da adolescência podem ser destacados. Para os itens de SE, os dados indicaram que os alunos tenderam a sentir-se insatisfeitos quanto à escola e não possuem boas percepções e expectativas desse espaço. Diversos aspectos do cotidiano escolar influenciam a avaliação que o estudante faz de sua escola. Fatores estruturais como o conforto térmico, acústico e qualidade da iluminação influenciam não só a aprendizagem, como também o bem-estar discente (Dalvite, Oliveira, Nunes, Perius, \& Scherer, 2007). Quanto à gestão, Vinha et al. (2018) destacam que boas percepções das normas, valores e das relações caracterizam-se como fator protetivo da violência escolar. Além disso, a SE está associada positivamente com a motivação para aprender, a autoconfiança para a realização de tarefas escolares e ao desenvolvimento emocional, coping, e suporte social (Blaya, Deabardieux, \& Vidal, 2004), desempenho escolar e as expectativas em relação ao futuro (Alves et al., 2015). Assim, torna-se essencial que a gestão da escola esteja mais próxima de seus alunos e identifiquem as principais causas de insatisfação dos estudantes e desenvolvem ações para saná-las.

No que diz respeito aos afetos, "cansado(a)" e "enturmado(a)" foram os itens mais endossados nos fatores de afetos negativos e positivos na escola; "compreendido(a)" e 
"furioso(o)" os menos assinalados. O cansaço é uma experiência subjetiva que envolve aspectos físicos, cognitivos e psicológicos (Cella \& Shalder, 2010). Considerando que a amostra desta pesquisa foi composta por adolescentes, sentir-se cansado é comum nesta etapa do desenvolvimento humano, considerando as mudanças físicas e biológicas. Além disso, o cansaço também pode estar relacionado à aspectos da realidade social do aluno e curricular da escola. Por tratar-se uma escola "modelo" da rede pública de ensino, possui grade curricular diferenciada, com maior número de atividades extracurriculares, captando alunos de municípios vizinhos, aumentando o percurso casa-escola (Conceição \& Zamora, 2015)

Sentir-se parte de um grupo, principalmente no contexto escolar, expressa uma característica típica do período da adolescência em que há um movimento de maior aproximação com os pares. Além disso, a escola constitui-se como um dos principais domínios de socialização (Chaves, 2015). Nesse contexto, a percepção de apoio social do estudante está associada à relação dos alunos com seus colegas e professores (Wang \& Eccles, 2012). O suporte social é um importante fator protetivo da saúde mental de adolescentes. Estudos evidenciam a sua importância na prevenção de sintomas depressivos e no uso abusivo de substâncias (Lardier, Barrios, Garcia-Reid, \& Reid, 2018; LorenzoBlanco, Unger, Oshri, Baezconde-Garbanati, \& Soto, 2016), além do engajamento escolar e comportamental (Coelho \& Dell'Áglio, 2018).

No entanto, cabe destacar que embora os alunos sintam-se inseridos socialmente na escola, os resultados indicaram ainda a falta de sentir-se compreendido. Tal dado, evidencia a importância ser trabalhada a qualidade das relações sociais no contexto escolar. A compreensão obtida no meio social é um importante componente do autoconceito e da formação da identidade do adolescente (Carvalho et al., 2017), além de caracterizar como uma variável protetiva contra a violência escolar (Giordani, Seffner, \& Dell'Aglio, 2017).

Ressalta-se ainda que o período da adolescência é marcado por sentimentos e atos de fúria, manifestando-se em comportamentos de indisciplina, vandalismo e incivilidade, principalmente no contexto escolar (Paluck, Sheperd, \& Aronow, 2016). Considerando o menor endosso do item furioso, evidencia um aspecto positivo da instituição, como um espaço saudável de respeito entre os sujeitos, como também um controle dos alunos em conter esse sentimento diante de situações estressores (Castro, Vieira, Moura, \& Lara, 2018). Ressalta-se a importância de que sejam fornecidos meios de interlocução entre alunos, professores e gestão pedagógica. Assim, mediante análise dos itens mais e menos endossados pelos participantes da amostra, observa-se um potencial uso da EBESE na produção de indicadores acerca de uma realidade escolar específica, possibilitando a utilização dos resultados para melhoria desse contexto.

No que diz respeito à consistência interna do instrumento, por meio de Teoria Clássica dos Testes e TRI, a EBESE apresentou coeficientes considerados adequados, embora os coeficientes obtidos pelo modelo de Rating Scale sejam mais baixos quando comparados aos valores de alfa e ômega. Este fato é justificável, pois no modelo Rating Scale é possível existir diferentes índices de precisão em determinados pontos da escala e o coeficiente de fidedignidade obtido por essa análise resulta da média desses diferentes índices de precisão, gerando uma taxa menor de resíduos e valores de coeficientes mais baixos. Ressalta-se que independente do modelo utilizado, os coeficientes de precisão do instrumento mantiveram-se dentro dos padrões. A partir das curvas de informação, constatou-se que o instrumento apresentou melhores índices de precisão em mensurar os alunos com baixos níveis de bem-estar subjetivo escolar, o que é considerado ideal, permitindo identificar estudantes para futuras intervenções. 
Esta pesquisa possibilitou o desenvolvimento de uma medida que auxiliará profissionais na área escolar, bem como avaliações educacionais de larga-escala. A investigação dos níveis de BES escolar dos estudantes favorecerá a identificação dos níveis de bem-estar do aluno na escola, bem como oportunizara-rá a possibilidade de melhoria do ambiente escolar. No entanto, apresenta algumas limitações, como a restrição da amostra a uma única escola. Além disso, não houve nenhum controle em relação aos tipos de resposta quando foram feitas as análises de estrutura interna do instrumento.

Van Vaerenbergh e Thomas (2013) apontam que estilos desejabilidade social e aquiescência podem impactar na correlação dos itens e, dessa forma, produzir vieses nos parâmetros de uma solução fatorial. Alguns estudos indicam a influencia desses vieses de resposta em instrumentos de autorrelato, principalmente em amostras de crianças e adolescentes (Soto, John, Gosling, \& Potter, 2008; Vigil-Colet, Fabia Morales-Vives, \& Lorenzo-Seva, 2013). Ressalta-se ainda a desejabilidade social como um viés que tem sido encontrado em medidas de autorrelato de BES (Caputo, 2017). Assim, seria interessante em pesquisas futuras a avaliação do impacto desses vieses de resposta na estrutura fatorial da EBESE, principalmente no que diz respeito à desejabilidade social, uma vez que o aluno em situação de avaliação da sua escola, pode se sentir desconfortável em responder ao instrumento. Além disso, destaca-se a importância de estudos serem realizados com amostras de diversas regiões do país para que os resultados sem representativos e generalizáveis.

\section{Referências}

Abed, A. L. Z. (2016). O desenvolvimento das habilidades socioemocionais como caminho para a aprendizagem e o sucesso escolar de alunos da educação básica. Construção Psicopedagógica, 24(25), 8-27.

Alves, C. F., Zappe, J. G., Patias, N. D., \& Dell'Aglio, D. D. (2015). Relações com a escola e expectativas quanto ao futuro em jovens brasileiros. Nuances: Estudos sobre educação, 26(1), 50-65. doi: 10.14572/nuances.v26i1.3818

Bardin, L.(2011). Análise de conteúdo. São Paulo: Edições 70.

Blaya, C., Debardieux, D., \& Vidal, D. (2004). Modos de organização da vida escolar nos estabelecimentos de ensino secundário inferior na Europa. Em C. Thelot (Ed.). Quel impact des politiques éducatives: Les apports de la recherche (pp. 127-154). Paris: Débat National sur L'avenir del'École.

Bond, T. G., \& Fox, C. M. (2015). Applying the rasch model fundamental measurement in the human sciences ( $3^{\text {rd }}$ ed.). Mahwah: Erlbaum.

Campo-Arias, A., \& Oviedo, H. C. (2008). Propriedades psicométricas de una escala: La consistencia interna. Salud Pública, 10(5), 831-839.

Caputo, A. (2017). Social desirability bias in self-reported well-being measures: Evidence from an online survey. Universitas Psychologica, 16(2), 245-255. doi: 10.11144/javeriana.upsy16-2.sdsw

Carneiro, C., \& Coutinho, L. G. (2015). Infância e adolescência: Como chegam as queixas escolares à saúde mental? Educar em Revista, 56, 181-192.

Carvalho, R. G., Fernandes, E., Câmara, J., Gonçalves, J. A., Rosário, J., Freitas, S., \& Carvalho, S. (2017). Relações de amizade e autoconceito na adolescência: Um estudo exploratório em contexto escolar. Estudos de Psicologia (Campinas), 34(3), 379388. doi:10.1590/1982-02752017000300006 
Castro, L. R., Vieira, C. B., Moura, I. K., \& Lara, J. S. (2018). Falas, afetos, sons e ruídos: As crianças e suas formas de habitar e participar do espaço escolar. Revista Eletrônica de Educação, 12(1), 151-168. doi: 10.14244/198271992019

Cella, M., \& Chalder, T. (2010). Measuring fatigue in clinical and community settings. Journal of Psychosomatic Research, 69(1), 17-22. doi: 10.1016/j.jpsychores.2009.10.007

Chaves, M. W. (2015). As relações entre a escola e o aluno: Uma história de em transformação. Educação \& Realidade, 40(4), 1149-1167. doi: 10.1590/2175623645958

Cintra, C. L., \& Guerra, V. M. (2017). Educação positiva: A aplicação da psicologia positiva à instituições educacionais. Psicologia Escolar e Educacional, 21(3), 505-514. doi: $10.1590 / 2175-35392017021311191$

Coelho, C. C. A., \& Dell'Áglio, D. D. (2018). Engajamento escolar: Efeito do suporte dos pais, professores e pares na adolescência. Psicologia Escolar e Educacional, 22(3), 621-629. doi: 10.1590/2175-35392018038539

Conceição, V. L., \& Zamora, M. H. R. N. (2015). Desigualdade social na escola. Estudos de Psicologia (Campinas), 32(4), 705-714. doi: 10.1590/0103-166X2015000400013

Dalvite, B., Oliveira, D., Nunes, G., Perius, M., \& Scherer, M. J. (2007). Análise do conforto acústico, térmico e lumínico em escolas da rede pública de Santa Maria, RS. Disciplinarum Scientia Artes, Letras e Comunicação, 8(1), 1-13.

Damásio, B. F. (2012). Uso da análise fatorial exploratória em psicologia. Avaliação Psicológica, 11(2), 213-228. Recuperado de http://pepsic.bvsalud.org/pdf/avp/v11n2/v11n2a07.pdf

Dias-Viana, J. L., \& Noronha, A. P. P. (2020). Bem-estar subjetivo de estudantes: Variáveis escolares associadas e medidas de avaliação. Manuscrito submetido para publicação.

Diener, E. (1984). Subjective well-being. Psychological Bulletin, 95(3), 542-575.

Diener, E. (2012). New findings and future directions for subjective well-being research. American Psychologist, 67(8), 590-597. doi: 10.1037/a0029541

Diener, E., Lucas, R. E., \& Oishi, S. (2005). Subjective well-being: The science of happiness and life satisfaction. Em: C. R. Snyder, \& S. J. Lopez (Eds.), Handbook of positive psychology (pp. 63-73). New York: Oxford University Press.

Giordani, J. P., Seffner, F., \& Dell'Aglio, D. D. (2017). Violência escolar: Percepções de alunos e professores de uma escola pública. Psicologia Escolar e Educacional, 21(1), 103-111. doi: 10.1590/2175-3539201702111092

Hair, J. F., Black, W. C., Babin, B. J., Anderson, R. E., Tatham, R. L. (2009). Análise multivariada de dados. Porto Alegre, RS: Artmed.

Hernández-Nieto, R. A. (2002). Contributions to statiscal analysis. Mérida: Universidad de los Andes.

Huebner, E. S. (1991). Initial development of the Student's Life Satisfaction Scale. School Psychology International, 12(3), 231-240. doi: 10.1177/0143034391123010

International Test Commission - ITC (2018). ITC guidelines for the large-scale assessment of linguistically and culturally diverse populations. Recuperado de https://www.intestcom.org/files/guideline_diverse_populations.pdf

King, A. L., Huebner, E. S., Suldo, S. M., \& Valois, R. F. (2006). An ecological view of school satisfaction in adolescence: Linkages between social support and behavior problems. Applied Research in Quality of Life, 1(3-4), 279-295. doi: $10.1007 / \mathrm{s} 11482-007-9021-7$ 
Konrath, S., Meier B. P, \& Bushman B. J. (2014) Development and validation of the Single Item Narcissism Scale (SINS). PLOS ONE, 9(8), e103469. doi: 10.1371/journal.pone.0103469

Lardier, D. T., Barrios, V. R., Garcia-Reid, P., \& Reid, R. J. (2018). Preventing substance use among hispanic urban youth: Valuing the role of family, social support networks, school importance, and community engagement. Journal of Child \& Adolescent Substance Abuse, 1-13. doi:10.1080/1067828x.2018.1466748

Linacre, J. M. (2014). Winsteps rash measurement computer program. Beaverton, OR: Winsteps.com. Recuperado de http://www.winsteps.com/index.htm

Lorenzo-Blanco, E. L., Unger, J. B., Oshri, A., Baezconde-Garbanati, L., \& Soto, D. (2016). Profiles of bullying victimization discrimination, social support, and school safety: Links with latino/a youth acculturation, gender, depressive symptons, and cigarette use. American Journal of Ortopsychiatry, 86(1), 37-48. doi: 10.1037/t03506-000

Lorenzo-Seva, U., Timmerman, M. E. \& Kiers, H. A. (2011). The hull method for selecting the number of common factors. Multivariate Behavioral Research, 46(2), 340-364. doi: 10.1080/00273171.2011.564527

McCullough, G., Huebner, S., \& Laughlin, J. E. (2000). Life events, self-concept, and adolescent's positive subjective well-being. Psychology in the Schools, 37(3), 281290. doi: 10.1002/(SICI)1520-6807(200005)37:3<281::AID-PITS8>3.0.CO;2-2

Ministério da Educação, Secretaria de Educação Básica, Secretaria de Educação Continuada, Alfabetização, Diversidade e Inclusão, Secretaria de Educação Profissional e Tecnológica, Conselho Nacional da Educação \& Câmara Nacional de Educação Básica (2013). Diretrizes curriculares nacionais gerais da educação básica. Recuperado http://portal.mec.gov.br/index.php?option=com_docman\&view=download\&alias $=1$ 3448-diretrizes-curiculares-nacionais-2013-pdf\&Itemid=30192

Ortuño-Sierra, J., Aritio-Solana, R., Luis, E. C. de., Nalda, F. N., \& Fonseca-Pedrero, E. (2017). Subjective well-being in adolescence: New psychometric evidences on the Satisfaction with Life Scale. European Journal of Developmental Psychology, 1-9. doi: 10.1080/17405629.2017.1360179

Paluck, E. L., Shepherd, H., \& Aronow, P. M. (2016). Changing climates of conflict: A social network experiment in 56 schools. Proceedings of the National Academy of Sciences, 113(3), 566-571. doi:10.1073/pnas.1514483113

Pasquali, L. (1999). Instrumentos psicológicos: Manual prático de elaboração. Brasília: LabPAM/ IBAPP.

Pasquali, L. (2001). Técnicas de exame psicológico (TPE): Manual - Fundamentos das técnicas psicológicas. São Paulo, SP: Casa do Psicólogo.

Reschly, A. L., Huebner, E. S., Appleton, J. J, \& Antaramian, S. (2008). Engagement as flourishing: The contribution of positive emotions and coping to adolescent's engagement at school and with learning. Psychology in the Schools, 45(5), 419-431. doi: 10.1002/pits.20306

Roeser, R. W. (2001). To cultivate the positive: Introduction to the special issue on schooling and mental health issues. Journal of School Psychology, 39, 99-110. doi: 10.1016/S0022-4405(01)00061-9

Sellstrom, E., \& Bremberg, S. (2006). Is there a "school effect" on pupil outcomes? A review of multilevel studies. Journal of Epidemiology \& Community Health, 60(2), 149-155. doi:10.1136/jech.2005.036707 
Soto, C. J., John, O. P., Gosling, S. D., \& Potter, J. (2008). The developmental psychometrics of big five self-reports: Acquiescence, factor structure, coherence, and differentiation from ages 10 to 20. Journal of Personality and Social Psychology, 94, 718-737. doi: 10.1037/0022-3514.94.4.718

Tian L., Wang D., \& Huebner, E. S. (2014). Development and validation of the Brief Adolescent's Subjective Well-Being in School Scale (BASWBSS). Social Indicators. Research, 120(2), 615-634. doi: 10.1007/s11205-014-0603-0

Van Vaerenbergh, Y., \& Thomas, T. D. (2013). Response styles in survey research: A literature review of antecedents, consequences, and remedies. International Journal of Public Opinion Research, 25(2), 195-217. doi: 10.1093/ijpor/eds021

Velicer, W. F. (1976). Determining the number of components from the matrix of partial correlations. Psychometrika, 41(3), 321-327.

Vigil-Colet, A., Morales-Vives, A., \& Lorenzo-Seva, U. (2013). How social desirability and acquiescence affect the age-personality relationship. Psicothema, 25(3), 342-348. doi: 10.7334/psicothema2012.297

Vinha, T. P., Tognetta, L. R. P., Azzi, R. G., Moro, A., Aragão, A. M. F. D., \& Morais, A. D. (2018). O clima escolar na perspectiva dos alunos de escolas públicas. Revista Educação e Cultura Contemporânea, 15(40), 163-186. doi: DOI10.5935/22381279.20180052PDF

Wang, M., \& Eccles, J. (2012). Social support matters: Longitudinal effects of social support on three dimensions of school engagement from middle to high school. Child Development, 83(3), 877-895. doi:10.1111/j.1467-8624.2012.01745.x

Whitley, A.M., Huebner, E.S., Hills, K.J., \& Valois, R.F. (2012). Can students be too happy in school? The optimal level of school satisfaction. Applied Research in Quality of Life, 7(4), 337-350. doi: 10.1007/s11482-012-9167-9

Financiamento: O presente trabalho foi realizado com apoio da Coordenação de Aperfeiçoamento de Pessoal de Nível Superior - Brasil (CAPES) - Código de Financiamento 001.

Participação dos autores: Participação dos autores: a) Planejamento e concepção do trabalho; b) Coleta de dados; c)Análise e interpretação de dados; d) Redação do manuscrito; e) Revisão crítica do manuscrito. J.L.D.V. contribuiu em a,b,c,d,e; A.P.P.N. em a,c,e.

Editora científica responsável: Dra. Cecilia Cracco 